\title{
Traduire
}

Revue française de la traduction

\section{Traduire, une lecture en profondeur}

\section{Michael Karpa}

\section{(2) OpenEdition \\ Journals}

Édition électronique

URL : http://journals.openedition.org/traduire/191

DOI : 10.4000/traduire.191

ISSN : 2272-9992

\section{Éditeur}

Société française des traducteurs

\section{Édition imprimée}

Date de publication : 1 juin 2011

Pagination : 62-69

ISSN : 0395-773X

\section{Référence électronique}

Michael Karpa, «Traduire, une lecture en profondeur », Traduire [En ligne], 224 | 2011, mis en ligne le 03 février 2014, consulté le 01 mai 2019. URL : http://journals.openedition.org/traduire/191 ; DOI :

10.4000/traduire.191 


\section{Traduire, une lecture en profondeur}

\section{Michael Karpa}

Fin 2008, comme beaucoup de collègues, le traducteur Michael Karpa connaît une chute brutale de la demande de traductions de ses plus gros clients. II se tourne alors vers des webséminaires, des organismes d'échanges d'informations et autres réseaux sociaux et formations en ligne pour l'utilisation d'outils de TAO, assiste à des rencontres et conférences d'auteurs débattant du rôle de l'Internet et comprend que l'impact de cet outil sur sa vie professionnelle, déjà essentiel, dépassera tout ce qu'il aurait pu imaginer.

L'insoutenable légèreté de la lecture. Nicholas Carr, dans son dernier livre The Shallows: What the Internet is Doing to Our Brains(1), étudie la façon dont l'internet modifie nos capacités cognitives et remodèle littéralement nos cerveaux malléables. D'autres - tel Malcolm Jones, journaliste à Newsweek, dans un article de juin 2010 Slow Reading: An Antidote for a Fast World?(2) - considèrent que nous lisons trop et trop vite. Mais N. Carr, s'appuyant largement sur la neurophysiologie et l'histoire, affirme que l'utilisation de l'Internet transforme la nature même de notre fonctionnement cognitif. Ainsi, l'auteur explique que nous lisons plus que jamais, mais nous ne faisons que survoler, passant de lien en lien, et consacrant beaucoup moins de temps à la lecture ou la pensée profondes. Pour Carr, " le Net semble réduire mes capacités de concentration et de réflexion ". Or, pour un traducteur, ce sont des aptitudes fondamentales. II s'agit en effet de comprendre chaque mot du document source, de percevoir le sens de l'ensemble du texte, d'en déduire certaines conclusions à partir d'autres données connues ou disponibles, puis de transmettre cette compréhension en reformulant l'original dans l'autre langue. Alors cette absence d'attention peut-elle nuire au traducteur que je suis ? À ceux qui ont besoin de la traduction ? À toute la profession?

(1) Carr Nicholas, The Shallows: What the Internet is Doing to Our Brains (Superficiel - Les effets de l'Internet sur notre cerveau). W.W. Norton \& Co., 2010.

(2) Jones Malcolm, "Slow Reading: An Antidote for a Fast World?" (L'antidote de la vitesse : la lecture) Newsweek, 23 juin 2010. 
Le traducteur en mutation ? L'Internet représente une véritable bénédiction pour le traducteur, les possibilités de recherche en ligne lui donnant très facilement accès à des connaissances spécialisées. J'ai d'abord eu du mal à croire que l'Internet puisse nuire à mes capacités de concentration. Puis, je me suis rappelé ce qui s'est passé lorsque j'ai découvert l'article du magazine The Atlantic ("Is Google Making Us Stupid?")(3) qui est à l'origine du livre de Carr : j'ai cliqué sur le lien, imprimé l'article, puis l'ai posé aussitôt sur une étagère - il était long, je n'avais pas le temps de m'y plonger. Lorsque je l'ai enfin lu, il m'a paru bien écrit, mais mon esprit était ailleurs dès la troisième page. L'auteur avait bien mis le doigt sur quelque chose. Puis j'ai eu la chance d'assister à un débat entre Carr et Peter Norvig, directeur de recherche chez Google, à San Francisco. N. Carr, auteur sans prétention, a par la suite accepté de répondre par courriel à mes questions concernant la traduction (The Shallows n'aborde pas la traduction et Carr indique clairement que ce sujet n'entre pas dans son domaine de compétences). Si N. Carr se voit souvent reprocher d'être anti-Internet, loin de maudire Internet lors de ce débat, l'auteur a expliqué qu'il s'était penché sur les modifications que subit la lecture et comment cela agit sur nos capacités cognitives. En lisant, nous utilisons d'abord une mémoire à court terme appelée mémoire de travail, qui retient nos impressions immédiates, nos sensations et nos pensées et forme, très concrètement, le contenu de notre conscience à un moment donné. La mémoire de travail " joue un rôle fondamental dans le transfert de l'information vers la mémoire à long terme et donc dans la création de nos connaissances propres " - lorsque la mémoire de travail s'efface, le travail semble neuf, comme écrit par quelqu'un d'autre(4). Mais l'internet surcharge systématiquement notre mémoire de travail en nous proposant de consulter une multitude de liens. Or, Carr cite plusieurs études de l'activité du lobe temporal indiquant que lorsque nous utilisons l'internet, le processus cognitif passe " de la lecture des mots aux jugements... [ce qui] on le sait perturbe la compréhension et la mémorisation, surtout lorsque cela est répété fréquemment. "Résultat, " notre capacité d'apprentissage en pâtit, et notre compréhension reste superficielle(5) ". Si l'utilisation de l'internet modifie physiquement notre cerveau - ce que Carr montre de façon plutôt convaincante - les capacités d'acquisition de vocabulaire et de réflexion approfondie du traducteur vont-elles se dégrader, privées de " la concentration créée par une lecture continue, attentive ... [qui] permet associations, déductions, conclusions, ainsi que la convocation d'analogies, pour aboutir à des solutions originales ? ". Concernant les effets éventuels des outils d'aide à la traduction sur la réflexion approfondie, Carr répond : " Je n'ai connaissance d'aucune étude sur les outils de traduction et leurs effets, mais selon une étude que je cite, plus un logiciel est utile pour

(3) Carr Nicholas, "Is Google Making Us Stupid?" (Google nous rend-il idiots ?) The Atlantic, Juillet/Août 2008.

(4) Ebrecht Ann, "Clear and Concise Writing," (Écrire avec clarté et concision) présentation lors de la 21e Conférence Internationale de traduction japonais/anglais, 24 avril 2010.

(5) Pour une critique des arguments de Carr et une défense passionnée des liens, voir Scott Rosenberg, "Delinkification is Bunk: Linking is Good for You," (Contre les Cassandre du lien).

www.salon.com/news/opinion/feature/2010/09/07/defending links open2010. 
nous aider à résoudre des problèmes, moins nous apprenons comment les résoudre nousmêmes ". Pour cette étude de 2003, le psychologue hollandais Christof van Nimwegen a soumis à un groupe des problèmes logiques complexes mais avec un logiciel conçu pour leur apporter une aide très efficace. Un autre groupe devait résoudre ces problèmes sans cette aide. Huit mois plus tard, van Nimwegen a constaté que le deuxième groupe était capable de résoudre les problèmes presque deux fois plus vite que le premier. Van Nimwegen en conclut que l'externalisation vers nos ordinateurs de tâches cognitives complexes réduit la capacité de notre cerveau à construire les structures cognitives réutilisables dans de nouvelles situations. Les traducteurs pourraient donc devenir dépendants des outils d'aide à la traduction, dictionnaires et autres aides en ligne au point de voir leurs capacités de traducteurs raisonnement, réflexion, compréhension - s'étioler faute d'être sollicitées ? Carr remarque que la correspondance écrite a perdu en expressivité avec le passage de l'écrit au courriel ou au texto et suggère de s'interroger sur la transformation de la syntaxe, question décisive pour les mémoires de traduction. Ces mémoires encouragent la traduction phrase par phrase et l'acceptation des correspondances à $100 \%$, l'adaptation de ces dernières au contexte devenant une étape superflue. On devine aisément le résultat d'une telle rigidité. Le désir de cohérence terminologique induit alors les clients à exiger une cohérence similaire pour les segments, puis les phrases entières. Les traducteurs risquent donc de devenir ce que Maryanne Wolf de l'Université Tufts appelle "de simples décodeurs d'information(6) " sans la capacité d'exploiter les " fertiles connexions mentales naissant d'une lecture approfondie ".

\section{Et le demandeur?}

De plus en plus, les traductions sont " consommées " via Internet. Les traductions produites par des systèmes comme Google Translate sont rarement consultées autrement. Si la réorganisation de nos cerveaux provoquée par l'internet complique la vie du traducteur, quel effet produit-elle sur nos clients, notre marché et nos lecteurs potentiels ? Les utilisateurs du net ont accès à des outils de traduction en ligne pour comprendre la teneur d'une page rédigée dans une langue qu'ils ignorent. En un clic, ils obtiennent une traduction suffisante pour un survol de 90 secondes permettant de passer au lien suivant. J'ai demandé à Carr si nous nous dirigions vers une norme de qualité dite "suffisante ". Si le survol est suffisant pour lire, une traduction maladroite ou partiellement inexacte pourrait devenir acceptable, autrement dit, la précision devient-elle superflue ? C'est très probable. "Une sorte de cercle vicieux est en marche, qui nous voit dépendre toujours plus de bases de données extérieures et d'outils de recherche, tandis que notre mémoire biologique se voit confier de moins en moins de connaissances, suscitant une dépendance encore accrue à ces outils externes ». Franz Och, respon-

(6) Wolf Maryanne, Proust and the Squid: The Story and Science of the Reading Brain (Proust et le calmar : histoire et effets de la lecture) Harper, 2007. 
sable des recherches sur la traduction machine pour Google, a donné une conférence le 30 juillet 2009, sur le développement de la traduction automatique(7), au cours de laquelle il a démontré l'amélioration de la qualité de celle-ci en comparant une traduction italien-anglais humaine avec la traduction automatique du même passage (cf. exemple 1) qu'il a demandé à son auditoire de distinguer, puis s'est étonné que certains y soient parvenus. En tant que traducteur ignorant l'italien, j'ai reconnu sans hésitation la version machine alors qu'Och les jugeait vraiment équivalentes. II semble que pour les utilisateurs d'Internet, la différence est négligeable, comme le suggère 0 ch. Et la machine est beaucoup plus rapide et moins chère. Va-t-on se contenter de l'à-peu-près, le produit fini devenu obsolète étant remplacé par une série d'itérations imparfaites ? Si la lecture-zapping devient la norme sur l'internet, l'escamotage de toute difficulté deviendra-t-il un élément de survie, voire une vertu?

\section{Et la profession ?}

Afin d'étudier plus précisément ce que cela implique pour la traduction professionnelle, je me suis penché sur Google Translate. Lors d'un entretien avec David Sarno dans le Los Angeles Times, Och dit de la traduction automatique qu'elle est " au cœur de la mission de Google(8) ". Selon le site officiel de Google (www.google.com/corporate) l'objectif de l'entreprise consiste en effet à organiser toute l'information disponible afin de la rendre accessible et utile au monde entier. Carr postule que pour Google, l'intelligence est le produit d'un processus mécanique qui peut être optimisé. II décrit Google comme un prolongement de l'époque de Frederick Winslow Taylor et des études du mouvement au début du xxe siècle, et cite Taylor : "Autrefois, l'homme avait la priorité ", mais " à l'avenir c'est le système qui l'aura ". Eric Schmidt, président de Google, a d'ailleurs déclaré que l'objectif de l'entreprise est de tout systématiser. Mais s'agissant de Google Translate, le processus pourrait bien ne pas être si mécanique qu'on l'a d'abord pensé. L'idée que le système ne sera jamais parfait est présente dès l'origine. Franz Och remarquait que " les langues sont très ambiguës [... et] le sens dépend du contexte... La traduction [automatique] suppose nombre de décisions. II faut faire des choix malgré l'incertitude ". La solution Google de décision statistique combine information floue et intégration d'une grande quantité de sources de données. La traduction automatique a connu deux approches. L'approche fonctionnelle décompose la langue en une série de règles sur lesquelles s'appuie le passage d'une langue à une autre. Cette solution ne fonctionne pas pour la traduction. Google a donc choisi d'utiliser sa puissance pour une approche statistique linéaire triant de nombreux modèles pour trouver la meilleure solution. Cela suppose un gigantesque corpus de traductions humaines dont l'élément central est le dictionnaire de phrases. À partir de ce vaste corpus, le système va trouver la traduction des mots d'une langue dans l'autre par les co-occurrences.

(7) Och Franz, www.youtube.com/watch?v=y PzPDRPwlA

(8) Sarno David (Los Angeles Times, 11 mars 2010). 
Ce faisant, il enregistre les phrases correspondantes et associe ces paires à une gradation en fonction du degré de correspondance. Google Translate fonctionne comme une bibliothèque qui extrapole : ses algorithmes cataloguent, associent et modifient le texte pour produire de nouvelles traductions à partir des précédentes. Mais de nombreux traducteurs ont fait l'expérience d'une association approuvée par le client dans une mémoire de traduction, mais fautive, même si chaque mot est correct. Le cerveau du traducteur reste donc irremplaçable. Ainsi, nous cesserions de générer le texte en langue d'arrivée pour en devenir les juges, autrement dit, correcteurs de textes générés par un ordinateur. La compréhension de plusieurs langues est bien sûr fondamentale pour la traduction. Prenons l'exemple des procès concernant la propriété intellectuelle et impliquant la " communication des pièces ", où les parties présentent d'énormes quantités de documents ; lorsqu'ils sont rédigés en langues étrangères, les traducteurs sont compétents pour se prononcer sur la pertinence de ces documents. À l'avenir, ce type d'intervention pourrait représenter une part importante de notre travail.

\section{De la nécessité du traducteur}

Les non-spécialistes pensent souvent que la traduction en ligne va rendre les traducteurs superflus. Carr se sert du parallèle avec l'écriture pour contrer l'argument en citant le Phèdre de Platon : Socrate craignait que le développement de l'écriture ne remplace dans l'esprit des gens des connaissances qu'ils mémorisaient auparavant. Leurs capacités mémorielles allaient donc décliner, et sans l'aptitude de comprendre les nouvelles informations à leur disposition, ils se croiraient savants sans l'être (cf. exemple 2). Pourtant, explique Carr, l'écriture et la lecture stimulent les idées neuves, diffusent l'information et étendent le savoir humain. Dans l'interview du Los Angeles Times, F. Och déclare : " Aujourd'hui, il faut pouvoir faire une recherche multilingue : taper une question, et si la réponse est une page web dans une langue étrangère, pouvoir la trouver et comprendre ce qu'elle contient ". Pour beaucoup, la traduction en ligne va permettre aux demandeurs potentiels de traduction de découvrir des informations intéressantes dans des langues qu'ils ne parlent pas, stimulant donc le besoin de faire traduire. D'ailleurs, les enquêtes montrent régulièrement que le marché se développe. Nombre d'avancées techniques s'emparent de la part la plus simple de notre travail, nous laissant la plus complexe, qui est aussi la plus satisfaisante. Tout comme les cabinets d'avocats disposent désormais de programmes tel l'eDiscovery d'IBM pour trier parmi les documents à communiquer ceux qui nécessitent une intervention humaine, le web pourrait limiter le travail du traducteur à ses aptitudes les plus pointues. Le volume d'information en constante augmentation et la vitesse de consultation, stimulée par la sollicitation de liens multiples sur l'internet, peuvent donner le vertige. Mais, selon Malcolm Jones de Newsweek: "Certes, nous sommes submergés d'informations, mais lire de plus en plus vite n'est manifestement pas la solution pour faire émerger les données pertinentes ". Se pourrait-il alors que le fondement 
de la traduction - la nécessité d'une compréhension exhaustive - décuple la valeur de nos compétences ? En effet, l'aptitude fondamentale du traducteur est précisément celle que le net fait disparaître : la lecture en profondeur.

Michael Karpa

\section{Parmi les travaux cités par N. Carr :}

JOHNSON Steven, Everything Bad is Good for You: How Today's Popular Culture is Actually Making Us Smarter (New York: Riverhead Books, 2005), www.stevenberlinjohnson.com.

KLINGBERG Torkel, The Overflowing Brain: Information Overload and the Limits of Working Memory, traduction Neil Betteridge (Oxford: Oxford University Press, 2009), www.oup.com/us/catalog/general/subject/Medicine/Neuroscience/ /dmlldz11c2EmY2k90Tc4 MDE5NTM3Mjg4NQ==.

PLATON, OEuvres (Garnier, Paris, 1919), (http://gallica.bnf.fr/ark:/12148/bpt6k57918430/f347.image)

SMALL G.W, MOODY T.D., SIDDARTH P., et BOOKHEIMER S.Y. Your Brain on Google: Patterns of Cerebral Activation During Internet Searching. American Journal of Geriatric Psychiatry, Vol. 17, n² 2 (février 2009) p. 116-26,

http://arunshanbhag.files.wordpress.com/2009/03/brainongoogle-amerjgp-2009.pdf.

SWELLER John, Instructional Design in Technical Areas (Camberwell, Australie: Australian Council for Educational Research, 1999).

TAYLOR Frederick Winslow, The Principles of Scientific Management (Harper \& Row, 1911), www.ibiblio.org/eldritch/fwt/ti.html.

VAN NIMWEGEN Christof, The Paradox of the Guided User: Assistance Can Be Counter-effective, SIKS Dissertation Series, № 2008-2009 (Université d'Utrecht, 31 mars 2008), http://en.scientificcommons.org/30004846.

VAN NIMWEGEN Christof, Herre van Oostendorp. "The Questionable Impact of an Assisting Interface on Performance in Transfer Situations. ॥ International Journal of Industrial Ergonomics, 39, n 3 (mai 2009), p. 501-508, http://portal.acm.org/citation.cfm?id=1362596. 


\section{Exemple 1 : Traductions italien -> anglais. Quelle est la version machine(9) ?}

\begin{tabular}{|c|c|}
\hline $\begin{array}{l}\text { NASA plans to send probe into Moon's sur- } \\
\text { face }\end{array}$ & $\begin{array}{l}\text { NASA's Plan to Send a Probe on the Lunar } \\
\text { Surface. }\end{array}$ \\
\hline $\begin{array}{l}\text { NASA, the United States' space agency, has } \\
\text { announced that its next mission to the Moon } \\
\text { will not only orbit the moon, but also send two } \\
\text { craft crashing into its surface. }\end{array}$ & $\begin{array}{l}\text { The American space agency, NASA, announced } \\
\text { that the next mission to the Moon will not be } \\
\text { limited to orbit around the satellite, but also } \\
\text { include the launch of two spacecraft that will } \\
\text { reach the lunar surface by means of a crash } \\
\text { landing. }\end{array}$ \\
\hline $\begin{array}{l}\text { The Lunar Reconnaissance Orbiter (LRO), } \\
\text { whose main mission pertains to mapping the } \\
\text { Moon, will send a spent fuel stage and an } \\
\text { impactor probe towards a crater on the South } \\
\text { Pole. }\end{array}$ & $\begin{array}{l}\text { The Lunar Reconnaissance Orbiter (LRO), } \\
\text { whose mission is to explore the Moon, will } \\
\text { send a shuttle to and support a probe into an } \\
\text { impact crater located on the south lunar pole. }\end{array}$ \\
\hline $\begin{array}{l}\text { The crater seems to be rich in hydrogen and } \\
\text { possibly ice. }\end{array}$ & $\begin{array}{l}\text { It seems that the crater is full of hydrogen and } \\
\text { possibly ice. }\end{array}$ \\
\hline $\begin{array}{l}\text { The mission is part of the run-up to trying to } \\
\text { land astronauts back on the Moon, and } \\
\text { perhaps keep them there for a longer period } \\
\text { of time than the Apollo missions did. }\end{array}$ & $\begin{array}{l}\text { This mission should be seen as part of a } \\
\text { series of initiatives that aim to bring the } \\
\text { astronauts on the moon and, perhaps, let us } \\
\text { stay for a period longer than the Apollo mission. }\end{array}$ \\
\hline
\end{tabular}

\section{Exemple 2 : Phèdre de Platon, traduction E. Chambry}

SOCRATE : C'est que l'écriture, Phèdre, a un grave inconvénient, tout comme la peinture. Les produits de la peinture sont comme s'ils étaient vivants ; mais pose-leur une question, ils gardent gravement le silence. II en est de même des discours écrits. On pourrait croire qu'ils parlent en personnes intelligentes, mais demande-leur de t'expliquer ce qu'ils disent, ils ne répondront qu'une chose, toujours la même. Une fois écrit, le discours roule partout et passe

(9) La NASA va envoyer une sonde sur la Lune. L'Agence spatiale américaine a annoncé que la prochaine mission lunaire ne se limitera pas à un vol en orbite autour de notre satellite mais prévoit aussi le lancement de deux véhicules qui atteindront le sol lunaire. Cette mission de reconnaissance (Lunar Reconnaissance Orbiter, LRO) destinée à l'exploration lunaire enverra une navette et une sonde à destination d'un cratère, situé au pôle sud lunaire, rempli d'hydrogène et peut-être de glace. Cette mission fait partie d'un programme en plusieurs volets destiné à envoyer des astronautes sur la Lune pour un séjour plus long que lors de la mission Apollo. 
indifféremment dans les mains des connaisseurs et dans celles des profanes, et il ne sait pas distinguer à qui il faut, à qui il ne faut pas parler. S'il se voit méprisé ou injurié injustement, il a toujours besoin du secours de son père ; car il n'est pas capable de repousser une attaque et de se défendre lui-même.

[Socrate poursuit à propos de ceux qui rédigent des discours écrits :] Si en composant ces ouvrages, ils ont connu la vérité, s'ils peuvent en venir à la discussion et défendre ce qu'ils ont écrit, et si l'orateur en eux est capable de faire pâlir l'auteur, ce n'est point leur activité d'écrivain, mais le souci de la vérité qui leur vaudra leur nom.

PHÈDRE : Quels sont les noms que tu leur donnes?

SOCRATE : Le nom de sage, Phèdre, me semble bien sublime et ne convient qu'à Dieu ; celui d'ami de la sagesse ou tel autre semblable leur conviendrait mieux et s'accorderait mieux à leur faiblesse.

(d'après un article paru dans l'ATA Chronicle, Revue de l'American Translators Association, janvier 2011, traduction Hélène Ladjadj)

Michael KARPA est traducteur (japonais $\rightarrow$ anglais), titulaire d'un Master's degree de politique internationale de l'Université de Stanford et a enseigné à l'école de traduction et d'interprétation du Monterey Institute of International Studies en 2002-2003. II a traduit What Is Japan de Sakaiya Taichi et ses essais et romans ont été publiés par Faultline et plusieurs autres magazines littéraires.

Contact:mskarpa@comcast.net. 